\title{
Risk Factors and Clavien-Dindo Classification of Postoperative Complications After Laparoscopic and Open Gastrectomies for Gastric Cancer: A Single-Center, Large Sample, Retrospective Cohort Study
}

\author{
Bo Lian* \\ Jie Chen* \\ Zhengyan $\mathrm{Li}$ \\ Gang Ji \\ Shiqi Wang \\ Qingchuan Zhao \\ Mengbin Li
}

Department of Digestive Surgery, National Clinical Research Center for Digestive Diseases, Xijing Hospital of Digestive Diseases, The Fourth Military Medical University, Xi'an, People's Republic of China

*These authors contributed equally to this work
This article was published in the following Dove Press journal: Cancer Management and Research

Background: Laparoscopy has been increasingly used for the surgery of gastric cancer. However, the postoperative complications are still under-investigated and the short-term results of laparoscopic gastrectomy remain controversial. This study aimed to explore the differences of postoperative complications between laparoscopic and open radical gastrectomies in patients with gastric cancer through the large sample size, retrospective cohort study, and evaluate the safety of laparoscopy in patients who underwent radical gastrectomy. Patients and Methods: A total of 2,966 patients with gastric cancer (TNM I III) who underwent laparoscopy or open gastrectomy from February 2009 to March 2016 were enrolled in this study. Complications were categorized according to the Clavien-Dindo classification. The incidence and severity of complications between laparoscopic and open gastrectomy were compared using one-to-three propensity score matching (PSM) analysis. Logistic regression analyses were performed to identify risk factors related to postoperative complications.

Results: A total of 2,966 patients were included in the study, including $687(23.2 \%)$ in the LG (Laparoscopy gastrectomies) group and 2,279 (76.8\%) in the OG (open gastrectomies) group. After PSM, a well-balanced cohort of 2,373 patients (676 cases in the LG group and 1,697 cases in the OG group) was further analyzed. The results showed that the incidence of overall complications in the LG group was significantly less than the OG group $(15.4 \%$ vs $20.8 \%, P=0.003$ ). However, the severe complications of the LG group showed no difference towards the OG group $(5.8 \%$ vs $5.8 \%, P=0.952)$. Multivariate analysis revealed that laparoscopic surgery is a protective factor for the reduction of postoperative complications. Age $\geq 60$ years, ASA classification IIIc and estimated blood loss $\geq 200 \mathrm{~mL}$ were confirmed as independent risk factors of overall complications.

Conclusion: Compared with traditional open gastrectomy, LG is safe and feasible with less trauma and fewer complications for patients with gastric cancer.

Keywords: gastric cancer, laparoscopy, gastric resection, postoperative complication, Clavien-Dindo classification

\section{Synopsis}

Laparoscopy has been increasingly used for the surgery of gastric cancer. However, the postoperative complications are still being investigated, and the short-term results of laparoscopic gastrectomy remain controversial. Although there have 
been many studies focused on the selection of the treatment option for gastric cancer, there are obvious constraints due to the limited sample size, race, social factors, or other factors. This study aimed to explore the difference between laparoscopic and open radical gastrectomies in the occurrence of postoperative complications of patients with gastric cancer through the large sample size, retrospective cohort study, and evaluate the safety of laparoscopy in patients undergoing radical gastrectomy, so as to bring a safer clinical decision.

\section{Introduction}

Gastric cancer is one of the most common malignant tumors around the world, and surgical resection is a very important clinical option for most patients. ${ }^{1}$ Some patients with early gastric cancer may only receive surgical treatment, but not follow-up comprehensive chemoradiotherapy. Now, laparoscopic gastrectomy (LG) has been increasingly performed for the treatment of gastric cancer, and the safety of laparoscopic surgery is the attended focus in minimally invasive surgeons. Laparoscopic surgery is performed with one or several small incisions and without direct vision. However, proper perigastric lymphadenectomy and R0 resection are needed to meet with the criteria for radical gastrectomy. Whether laparoscopic gastrectomy could achieve comparable efficacy as open gastrectomy for gastric cancer and the postoperative complications in the short-term are still under investigation. Clavien-Dindo classification system ${ }^{2}$ is the most credible standard for evaluating postoperative complications including laparoscopic radical gastrectomy for gastric cancer. This study retrospectively analyzed the clinicopathologic data of 2,966 patients who received radical gastrectomy for gastric cancer in our center, the overall complications and serious complications after radical gastrectomy by laparoscopy and laparotomy were compared, and the risk factors of postoperative complications were analyzed.

\section{Patients and Methods}

\section{Patients}

The clinicopathological data of 2,966 patients who underwent radical gastrectomy for gastric cancer in the digestive surgery department of our hospital from February 2009 to March 2016 were retrospectively collected and analyzed. Only those who were pathologically diagnosed with gastric cancer and received laparotomy or laparoscopic radical gastrectomy for gastric cancer in our hospital were included. Pathologic staging of gastric cancer was based on the 8th edition of the Union for International Cancer Control (UICC) tumor staging criteria. ${ }^{3}$ Perigastric lymph node dissection was performed after classification and sent for pathological examination. The inclusion criteria for this study were as follows: pathologically diagnosed with gastric adenocarcinoma, pT1-T4a, pN0-N3, M0; patients aged 18-75 years old; received laparotomy or laparoscopic radical gastrectomy for gastric cancer; not combined with severe systemic disease (such as inflammatory bowel disease, severe cardiopulmonary disease, diabetes, severe hepatic, and renal dysfunction); no need of emergency surgery; no history of gastric cancer surgery or adjuvant chemotherapy; not combined with other malignant lesions; and not combined with other vital viscera resection. Finally, a total of 2,966 patients were included in this study. Patients were divided into a laparoscopic group (LG) and open operation (OG) group according to the operation mode. There were 687 patients in the laparoscopic group and 2,279 patients in the laparotomy group. As the total number of patients in the OG group was about 3-times higher than that in LG Group, a 1:3 propensity score matching was conducted according to age, gender, ASA classification, gastric resection range, tumor differentiation, TNM stage, BMI, tumor size, and other baseline data. After matching, a total of 2,373 patients met the inclusion and exclusion criteria, 676 patients in the LG group and 1,697 patients in the OG group. Baseline data of patients are shown in Table 1. This study was approved by the Institutional Review Board of our hospital, and written informed consent was obtained from all the patients prior to surgery.

\section{Surgery Procedures}

The basis for determining the surgical plan was preoperative CT scan findings of cancer, systemic function evaluation of patients, the experience of the operator, and willingness of the patients. All patients signed the informed consent form before the operation, which was completed by the experienced digestive surgery team of our hospital. The extent of gastric resection was decided according to the size and location of tumor, based on the 4th edition of Japanese guidelines for the treatment of gastric cancer. ${ }^{4}$ Proximal and distal gastrectomy were defined as partial gastrectomy when risk factor analysis was conducted. All the included cases were up to R0 resection. Specific surgical procedures have been described in the previous studies of our center. ${ }^{5,6}$ Patients 
Table I The Demographic Characteristics of the Patients

\begin{tabular}{|c|c|c|c|c|c|c|c|c|}
\hline & \multicolumn{3}{|l|}{ Before PSM } & \multirow[t]{2}{*}{$P$-value } & \multicolumn{3}{|l|}{ After PSM } & \multirow[t]{2}{*}{$P$-value } \\
\hline & $\begin{array}{l}\text { Entire, } \\
\mathrm{n}=2,966(100)\end{array}$ & $\begin{array}{l}\text { LG, } n= \\
687(23.2)\end{array}$ & $\begin{array}{l}\text { OG, } \\
n=2,279(76.8)\end{array}$ & & $\begin{array}{l}\text { Entire } \\
2373(100)\end{array}$ & $\begin{array}{l}\text { LG } 676 \\
(28.5)\end{array}$ & $\begin{array}{l}\text { OG } \\
1697(71.5)\end{array}$ & \\
\hline Age (years) (mean $\pm S D)$ & $57.4 \pm 9.8$ & $57.1 \pm 9.5$ & $57.5 \pm 9.8$ & 0.412 & $57.2 \pm 9.7$ & $57.2 \pm 9.5$ & $57.2 \pm 9.8$ & 0.981 \\
\hline $\begin{array}{l}\text { Gender } \\
\text { Male } \\
\text { Female }\end{array}$ & $\begin{array}{l}2,24 \mathrm{I}(75.6) \\
725(24.4)\end{array}$ & $\begin{array}{l}532(77.4) \\
155(22.6)\end{array}$ & $\begin{array}{l}\text { I,709 (75.0) } \\
570(25.0)\end{array}$ & 0.193 & $\begin{array}{l}\mathrm{I}, 825(76.9) \\
548(23 . \mathrm{I})\end{array}$ & $\begin{array}{l}522(77.2) \\
154(22.8)\end{array}$ & $\begin{array}{l}\text { I,303 (76.8) } \\
394(23.2)\end{array}$ & 0.820 \\
\hline $\begin{array}{l}\text { ASA } \\
\text { I } \\
\text { II } \\
\text { III }\end{array}$ & $\begin{array}{l}|3|(4.4) \\
2,468(83.2) \\
367(12.4)\end{array}$ & $\begin{array}{l}32(4.7) \\
579(84.3) \\
76(11.1)\end{array}$ & $\begin{array}{l}99(4.3) \\
1,889(82.9) \\
291(12.7)\end{array}$ & 0.476 & $\begin{array}{l}\text { III (4.7) } \\
\text { I,988 (83.8) } \\
274(\text { II.5) }\end{array}$ & $\begin{array}{l}30(4.4) \\
570(84.3) \\
76(11.2)\end{array}$ & $\begin{array}{l}8 \mathrm{I}(4.8) \\
\mathrm{I}, 4 \mathrm{I} 8(83.6) \\
198(\mathrm{II} .7)\end{array}$ & 0.894 \\
\hline $\begin{array}{l}\text { Resection extent } \\
\text { Proximal } \\
\text { Distal } \\
\text { Total }\end{array}$ & $\begin{array}{l}26 \mid(8.8) \\
I, 23 \mid(4 \mid .5) \\
I, 474(49.7)\end{array}$ & $\begin{array}{l}40(5.8) \\
305(44.5) \\
342(49.8)\end{array}$ & $\begin{array}{l}221(9.7) \\
926(40.6) \\
I, 132(49.7)\end{array}$ & 0.004 & $\begin{array}{l}154(6.5) \\
989(41.7) \\
1,230(51.8)\end{array}$ & $\begin{array}{l}39(5.8) \\
297(43.9) \\
340(50.3)\end{array}$ & $\begin{array}{l}\text { II } 5(6.8) \\
692(40.8) \\
890(52.4)\end{array}$ & 0.310 \\
\hline $\begin{array}{l}\text { Digestive tract } \\
\text { reconstruction } \\
\text { Billroth I } \\
\text { Billroth II } \\
\text { Roux-en-Y } \\
\text { Esophagogastrostomy }\end{array}$ & $\begin{array}{l}346(11.7) \\
882(29.7) \\
1,479(49.9) \\
259(8.7)\end{array}$ & $\begin{array}{l}52(7.6) \\
25 I(36.5) \\
343(49.9) \\
4 I(6.0)\end{array}$ & $\begin{array}{l}294(12.9) \\
631(27.7) \\
1,136(49.8) \\
218(9.6)\end{array}$ & $<0.001$ & $\begin{array}{l}196(8.3) \\
789(33.2) \\
1,234(52.0) \\
154(6.5)\end{array}$ & $\begin{array}{l}52(7.7) \\
244(36.1) \\
34 I(50.4) \\
39(5.8)\end{array}$ & $\begin{array}{l}\text { I } 44(8.5) \\
545(32.1) \\
893(52.6) \\
\text { II }(6.8)\end{array}$ & 0.276 \\
\hline $\begin{array}{l}\text { Histological } \\
\text { differentiation } \\
\text { Well/mod } \\
\text { Poor } \\
\text { Sig/muc }\end{array}$ & $\begin{array}{l}\mathrm{I}, 035(34.9) \\
\mathrm{I}, 825(6 \mathrm{I} .5) \\
\mathrm{I} 06(3.6)\end{array}$ & $\begin{array}{l}277(40.3) \\
383(55.7) \\
27(3.9)\end{array}$ & $\begin{array}{l}748(32.8) \\
\mathrm{I}, 446(63.4) \\
85(3.7)\end{array}$ & $<0.001$ & $\begin{array}{l}896(37.8) \\
1,394(58.7) \\
83(3.5)\end{array}$ & $\begin{array}{l}275(40.7) \\
379(56.1) \\
22(3.2)\end{array}$ & $\begin{array}{l}62 \mathrm{I}(36.6) \\
\mathrm{I}, 0 \mathrm{I} 5(59.8) \\
6 \mathrm{I}(3.6)\end{array}$ & 0.178 \\
\hline $\begin{array}{l}\text { PT stage } \\
\text { TI } \\
\text { T2 } \\
\text { T3 } \\
\text { T4a }\end{array}$ & $\begin{array}{l}55 I(18.6) \\
399(13.5) \\
998(33.6) \\
I, 018(34.3)\end{array}$ & $\begin{array}{l}I 5 I(22.0) \\
112(16.3) \\
234(34.1) \\
190(27.7)\end{array}$ & $\begin{array}{l}400(17.6) \\
287(12.6) \\
764(33.5) \\
828(36.3)\end{array}$ & $<0.001$ & $\begin{array}{l}467(19.7) \\
338(14.2) \\
847(35.7) \\
721(30.4)\end{array}$ & $\begin{array}{l}144(21.3) \\
110(16.3) \\
233(34.5) \\
189(28.0)\end{array}$ & $\begin{array}{l}323(19.0) \\
228(13.4) \\
614(36.2) \\
532(31.3)\end{array}$ & 0.101 \\
\hline $\begin{array}{l}\mathrm{pN} \text { stage } \\
\text { N0 } \\
\mathrm{N} 1 \\
\mathrm{~N} 2 \\
\mathrm{~N} 3 \mathrm{a} \\
\mathrm{N} 3 \mathrm{~b}\end{array}$ & $\begin{array}{l}I, 042(35.1) \\
518(17.5) \\
54 I(18.2) \\
518(17.5) \\
347(11.7)\end{array}$ & $\begin{array}{l}277(40.3) \\
118(17.2) \\
116(16.9) \\
118(17.2) \\
58(8.4)\end{array}$ & $\begin{array}{l}765(33.6) \\
400(17.6) \\
428(18.6) \\
400(17.6) \\
289(12.7)\end{array}$ & 0.003 & $\begin{array}{l}892(37.6) \\
416(17.5) \\
421(17.7) \\
430(18.1) \\
214(9.0)\end{array}$ & $\begin{array}{l}269(39.8) \\
116(17.2) \\
116(17.2) \\
118(17.5) \\
57(8.4)\end{array}$ & $\begin{array}{l}623(36.7) \\
300(17.1) \\
305(18.0) \\
312(18.4) \\
157(9.3)\end{array}$ & 0.724 \\
\hline $\begin{array}{l}\text { PTNM stage } \\
\text { I } \\
\text { II } \\
\text { III }\end{array}$ & $\begin{array}{l}699(23.6) \\
819(27.6) \\
1,448(48.8)\end{array}$ & $\begin{array}{l}191(27.8) \\
203(29.5) \\
293(42.6)\end{array}$ & $\begin{array}{l}508(22.3) \\
616(27.0) \\
I, 155(50.7)\end{array}$ & 0.001 & $\begin{array}{l}598(25.2) \\
687(29.0) \\
\mathrm{I}, 088(45.8)\end{array}$ & $\begin{array}{l}184(27.2) \\
200(29.6) \\
292(43.2)\end{array}$ & $\begin{array}{l}414(24.4) \\
487(28.7) \\
796(46.9)\end{array}$ & 0.211 \\
\hline BMI $\left(\mathrm{kg} / \mathrm{m}^{2}\right)($ mean $\pm \mathrm{SD})$ & $22.3 \pm 3.2$ & $22.4 \pm 3.3$ & $22.3 \pm 3.2$ & 0.473 & $22.4 \pm 3.2$ & $22.4 \pm 3.3$ & $22.4 \pm 3.2$ & 0.787 \\
\hline $\begin{array}{l}\text { Tumor size }(\mathrm{cm}) \\
(\text { mean } \pm S D)\end{array}$ & $4.4 \pm 2.5$ & $4.2 \pm 2.6$ & $4.4 \pm 2.5$ & 0.011 & $4.3 \pm 2.4$ & $4.2 \pm 2.7$ & $4.3 \pm 2.3$ & 0.286 \\
\hline $\begin{array}{l}\text { Postoperative hospital } \\
\text { stay (days) (mean } \pm S D \text { ) }\end{array}$ & $8.1 \pm 4.0$ & $8.0 \pm 3.5$ & $8.1 \pm 4.1$ & 0.599 & $8.1 \pm 4.0$ & $8.0 \pm 3.5$ & $8.1 \pm 4.2$ & 0.522 \\
\hline
\end{tabular}

(Continued) 
Table I (Continued).

\begin{tabular}{|c|c|c|c|c|c|c|c|c|}
\hline & \multicolumn{3}{|l|}{ Before PSM } & \multirow[t]{2}{*}{$P$-value } & \multicolumn{3}{|l|}{ After PSM } & \multirow[t]{2}{*}{$P$-value } \\
\hline & $\begin{array}{l}\text { Entire, } \\
n=2,966(100)\end{array}$ & $\begin{array}{l}\text { LG, } n= \\
687(23.2)\end{array}$ & $\begin{array}{l}\text { OG, } \\
n=2,279(76.8)\end{array}$ & & $\begin{array}{l}\text { Entire } \\
2373(100)\end{array}$ & $\begin{array}{l}\text { LG } 676 \\
(28.5)\end{array}$ & $\begin{array}{l}\text { OG } \\
\mid 697(71.5)\end{array}$ & \\
\hline $\begin{array}{l}\text { Operation time }(\mathrm{min}) \\
(\operatorname{mean} \pm S D)\end{array}$ & $206.2 \pm 68.3$ & $266.1 \pm 69.7$ & $188.2 \pm 56.5$ & $<0.001$ & $211.3 \pm 70.3$ & $\begin{array}{l}266.2 \\
\pm 70.2\end{array}$ & $|89.4 \pm 57|$. & $<0.001$ \\
\hline $\begin{array}{l}\text { Number of retrieved } \\
\text { lymph (mean } \pm S D)\end{array}$ & $27.0 \pm 8.9$ & $24.8 \pm 7.0$ & $27.6 \pm 9.3$ & $<0.001$ & $26.7 \pm 8.6$ & $24.8 \pm 7.0$ & $27.4 \pm 9.1$ & $<0.001$ \\
\hline $\begin{array}{l}\text { Estimate blood loss }(\mathrm{mL}) \\
(\text { mean } \pm \mathrm{SD})\end{array}$ & $200.9 \pm 161.6$ & $\begin{array}{l}180.6 \\
\pm 171.3\end{array}$ & $207.0 \pm 158.1$ & $<0.001$ & $\begin{array}{l}199.7 \\
\pm 152.9\end{array}$ & $\begin{array}{l}180.8 \\
\pm 172.0\end{array}$ & $\begin{array}{l}205.9 \\
\pm 144.0\end{array}$ & $<0.001$ \\
\hline
\end{tabular}

Abbreviations: ASA, American Society of Anesthesiologists; BMI, body mass index; T, tumor; N, node; M, metastasis; TNM, tumor node metastases; SD, standard deviation; Well/mod, well/moderately differentiated.

with advanced gastric cancer routinely received adjuvant chemotherapy based on 5-fluorouracil.

\section{Assessment Criteria}

The primary indicators were the incidence, severity of postoperative complications and then the risk factors to postoperative complications. Complications were defined within 30 days after surgery. The postoperative complications categories were classified according to consensus formulated by the international gastric cancer association and the Japanese gastric cancer association in 2018 (Japanese gastric cancer treatment guidelines 2014 (ver.4)). ${ }^{7}$ The severity of postoperative complications was assessed by Clavien-Dindo classification system $^{2,8}$ and divided into overall complications and severe complications. Those Clavien-Dindo classifications above grade wa were defined as severe complications in this study. Patients who had a variety of complications were graded according to the most severe complications.

The research process is shown in Figure 1.

\section{Statistical Analysis}

Statistical analysis was performed by SPSS 24 software (IBM, New York). Quantitative data were expressed as mean \pm standard deviation (SD), using the one-way ANOVA test ( $F$-test). Counting data was represented by cases and percentages. The Chi-squared test was performed when comparing the frequencies of categorical data. A one-to-one propensity score matching was conducted using SPSS PSMMATHING 3.04 plug-in and R 3.20 program, with a caliper width set at 0.02 , and nearest neighbor matching only and without replacement principle was adopted. Univariate analysis of risk factors for postoperative complications chi-square test, logistic regression model was applied for multivariate analysis. A $P$-value $<0.05$ was considered as statistically significant.

\section{Results}

\section{Clinical Features and Surgical Outcomes}

As shown in Table 1, we selected 2,966 patients who underwent radical gastrectomy in our center from February 2009 to March 2016. After PSM, a total of 2,373 patients met the inclusion and exclusion criteria, 676 patients in the LG group and 1,697 patients in the OG group. Of the 2,373 patients included in this study, $1,825(76.9 \%)$ were male and $548(23.1 \%)$ were female. There was no significant difference in age, gender, preoperative ASA, gastric resection extent, histological differentiation, pTNM stage, preoperative BMI, and other baseline data between the two groups ( $P$-value $>0.05$ ). As shown in Table 1, the postoperative hospitalization was comparable in the $\mathrm{LG}$ and traditional $\mathrm{OG}$ groups ( $8.0 \pm 3.5$ vs $8.1 \pm 4.2$ days, $P=0.522)$, However, a longer operation time was needed for LG than that of OG $\quad(266.2 \pm 70.2$ minutes vs $189.4 \pm 57.1$ minutes, $P<0.001$ ), a lower number of lymph nodes retrieved in the LG than in the OG $(24.8 \pm 7.0$ vs $27.4 \pm 9.1, P<0.001)$ group, and less estimated blood loss in the LG group than the OG group $(180.8 \pm 172.0 \mathrm{~mL}$ vs 205.9 $\pm 144.0 \mathrm{~mL}, P<0.001)$. No difference was observed when comparing the severe complications in the two groups $(5.80 \%$ vs $5.8 \%, 0.952)$ (Table 2$)$. 


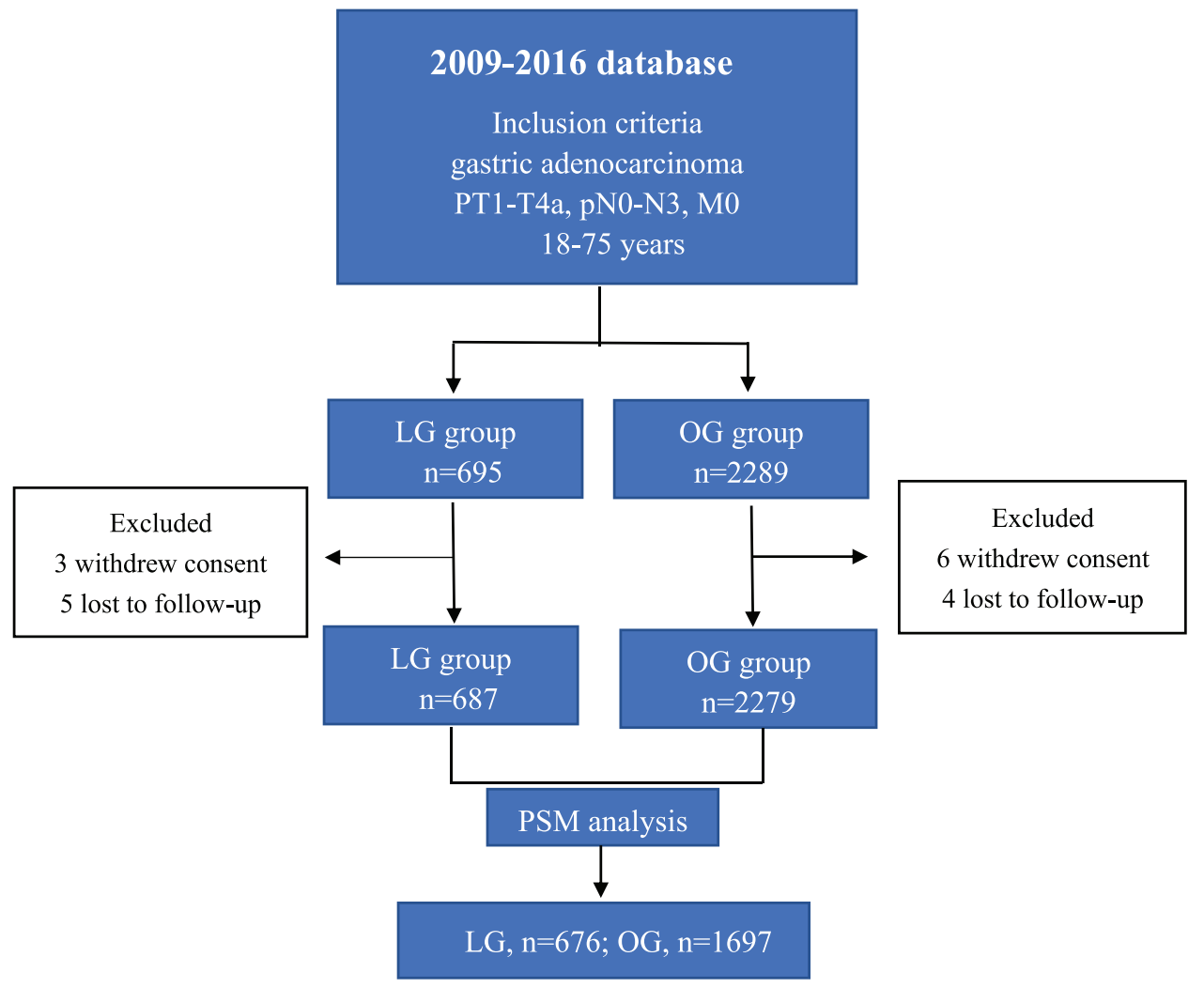

Figure I Research process.

\section{Complications Assessed by Clavien- Dindo Classification System}

Table 3 shows the complications assessed using the Clavien-Dindo classification system. The mild complications in the LG group were significantly less than the OG group Clavien-Dindo classification sgrade II) (18.2\% vs $11.2 \%, P<0.001)$. The rate of overall postoperative complications showed the same pattern as minor complications in both groups. There was one patient in the OG group who died from intestinal adhesion and perforation, and another experienced respiratory and cardiac arrest, rescues the invalid death in the OG group, no patient died in the LG group. There was no statistically significant difference in postoperative mortality between the two groups $(P=0.913)$.

\section{Subgroup Analysis of Postoperative Complications}

Subgroup analysis was conducted according to clinical features of the patients, considering the overall complications, except in subgroups of female, ASA grade ш, TNM stage $\mathrm{I}, \mathrm{BMI} \geq 24 \mathrm{~kg} / \mathrm{m}^{2}$, distal gastrectomy, tumor size

Table 2 Complications of the Two Groups

\begin{tabular}{|c|c|c|c|c|}
\hline & LG & OG & \multirow[t]{2}{*}{ Statistic } & \multirow[t]{2}{*}{ P-value } \\
\hline & $n=676(28.5)$ & $n=I, 697(7 \mid .5)$ & & \\
\hline $\begin{array}{l}\text { Overall complication } \\
\text { Yes } \\
\text { No }\end{array}$ & $\begin{array}{l}104(15.4) \\
572(4.6)\end{array}$ & $\begin{array}{l}353(20.8) \\
1,344(79.2)\end{array}$ & $\chi^{2}=9.122$ & 0.003 \\
\hline $\begin{array}{l}\text { Severe complications } \\
\text { Yes } \\
\text { No }\end{array}$ & $\begin{array}{l}39(5.8) \\
637(94.2)\end{array}$ & $\begin{array}{l}99(5.8) \\
\mathrm{I}, 598(94.2)\end{array}$ & $\chi^{2}=0.003$ & 0.952 \\
\hline
\end{tabular}


Table 3 Postoperative Complications Assessed by ClavienDindo Classification System

\begin{tabular}{|c|c|c|c|}
\hline Variables & $\begin{array}{l}\text { LG Group, } \\
\text { n (\%) }\end{array}$ & $\begin{array}{l}\text { OG Group, } \\
\text { n (\%) }\end{array}$ & $P$ \\
\hline$\leq \mathrm{II}$ & $55(8.1)$ & $254(15.0)$ & 0.001 \\
\hline Fever $\left(>38^{\circ} \mathrm{C}\right)$ & $2(0.3)$ & $8(0.5)$ & 0.807 \\
\hline Nausea and vomiting & $0(0)$ & $3(0.2)$ & 0.650 \\
\hline $\begin{array}{l}\text { Transient hepatic } \\
\text { function damage }\end{array}$ & $\mathrm{I}(0.1)$ & $4(0.2)$ & 1.000 \\
\hline Wound complications & $9(1.3)$ & $5 I(3.0)$ & 0.019 \\
\hline $\begin{array}{l}\text { Intra-abdominal } \\
\text { infection }\end{array}$ & $3(0.4)$ & $13(0.8)$ & 0.557 \\
\hline Pneumonia & $13(1.9)$ & 91 (5.4) & $<0.001$ \\
\hline Anemia & $14(2.1)$ & $36(2.1)$ & 0.939 \\
\hline Pleural effusion & $3(0.4)$ & $9(0.5)$ & 1.000 \\
\hline Anastomosis bleeding & $\mathrm{I}(0.1)$ & $3(0.2)$ & 1.000 \\
\hline Bowel obstruction & $\mathrm{I}(0.1)$ & $9(0.5)$ & 0.344 \\
\hline Hypoproteinemia & $2(0.3)$ & $8(0.5)$ & 0.807 \\
\hline Leakage of lymphatics & $3(0.4)$ & $8(0.5)$ & 1.000 \\
\hline Anastomosis stenosis & $\mathrm{I}(0.1)$ & $2(0.1)$ & 1.000 \\
\hline Intra-abdominal bleeding & $\mathrm{I}(0.1)$ & $2(0.1)$ & 1.000 \\
\hline Retention of urine & $0(0)$ & $\mathrm{I}(0.1)$ & 1.000 \\
\hline Intraperitoneal effusion & $\mathrm{I}(0.1)$ & $6(0.4)$ & 0.679 \\
\hline ша & $23(3.4)$ & $48(2.8)$ & 0.411 \\
\hline Pleural effusion & $6(0.9)$ & $\mathrm{II}(0.6)$ & 0.723 \\
\hline Intra-abdominal bleeding & $2(0.3)$ & $4(0.2)$ & 1.000 \\
\hline Wound complications & $6(0.9)$ & $12(0.7)$ & 0.647 \\
\hline Intraperitoneal effusion & $3(0.4)$ & $4(0.2)$ & 0.671 \\
\hline Anastomosis leakage & $\mathrm{I}(0.1)$ & $5(0.3)$ & 0.850 \\
\hline Anastomosis bleeding & $2(0.3)$ & $5(0.3)$ & 1.000 \\
\hline $\begin{array}{l}\text { Intra-abdominal } \\
\text { infection }\end{array}$ & $3(0.4)$ & $7(0.4)$ & 1.000 \\
\hline шb & $15(2.2)$ & $4 \mathrm{I}(2.4)$ & 0.775 \\
\hline Wound problem & $\mathrm{I}(0.1)$ & $11(0.6)$ & 0.219 \\
\hline $\begin{array}{l}\text { Intra-abdominal } \\
\text { infection }\end{array}$ & $0(0)$ & $6(0.4)$ & 0.273 \\
\hline Anastomosis bleeding & $2(0.3)$ & $5(0.3)$ & 1.000 \\
\hline Intra-abdominal bleeding & $\mathrm{I}(0.1)$ & $4(0.2)$ & 1.000 \\
\hline Intestinal obstruction & $5(0.7)$ & $10(0.6)$ & 0.898 \\
\hline Pancreatitis & $3(0.4)$ & $\mathrm{I}(0.1)$ & 0.131 \\
\hline $\begin{array}{l}\text { Deep venous } \\
\text { thrombosis }\end{array}$ & $\mathrm{I}(0.1)$ & $0(0)$ & 0.634 \\
\hline Anastomotic fistula & $2(0.3)$ & $4(0.2)$ & 1.000 \\
\hline IVa & $0(0)$ & $8(0.5)$ & 0.163 \\
\hline Heart failure & $0(0)$ & $3(0.2)$ & 0.650 \\
\hline Lung failure & $0(0)$ & $4(0.2)$ & 0.478 \\
\hline Renal failure & $0(0)$ & $\mathrm{I}(0.1)$ & 1.000 \\
\hline $\mathrm{IVb}$ & $\mathrm{I}(0.1)$ & $\mathrm{I}(0.1)$ & 1.000 \\
\hline Multiple organ failure & $\mathrm{I}(0.1)$ & $\mathrm{I}(0.1)$ & 1.000 \\
\hline$V$ (Death) & $0(0)$ & $2(0.1)$ & 0.913 \\
\hline
\end{tabular}

$<5 \mathrm{~cm}$, operation time $\leq 180$ minutes, and the number of retrieved lymph nodes $<25$. There were significant differences between the two groups in most of the subgroups, as shown in Table 4. However, within the severe complications, statistical differences between the LG and OG group were present only in stratifications of number of retrieved lymph nodes $\geq 25$, whereas no difference was found in the rest of the stratifications, as shown in Table 5.

\section{Univariate and Multivariate Analyses of Risk Factors for Postoperative Complications}

Risk factors associated with postoperative complications following LG and OG for gastric cancer in the wellmatched cohort were determined using logistic regression. Univariate analysis showed that age, gastric resection extent, ASA, tumor size, T stage, TNM stage, estimated blood loss and operation method were correlated with the overall postoperative complications, and age, gastric resection extent, tumor size, T stage, TNM stage, and estimate blood loss were correlated with severe postoperative complications. Of these, multivariate analysis revealed that laparoscopic operation was a protective factor for reduced postoperative complications $(P=0.003)$; age $\geq 60$ years, ASA grade $ш$, and estimated blood loss $\geq 200 \mathrm{~mL}$ were independent risk factors for overall complications, and age $\geq 60$ years, ASA grade $ш$, pT2-T4a stage, and estimated blood loss $\geq 200 \mathrm{~mL}$ were independent risk factors for severe complications. Detailed data are shown in Table 6 .

\section{Discussion}

So far, laparoscopy has been increasingly used in the surgical treatment of gastric cancer., ${ }^{5,-6,-9-12}$

The safety of laparoscopy is the focus of minimally invasive surgeons. The Clavien-Dindo classification system is a universally accepted evaluation standard of postoperative complications. ${ }^{2,8,13,14}$ The results of this study showed that laparoscopic radical gastrectomy for gastric cancer was safe and feasible, and the overall postoperative complications and intraoperative estimate blood loss were lower than those of laparotomy, in terms of better minimally invasive effect. Korean scholars Kim et al ${ }^{15}$ reported that there was no statistically significant difference in the incidence and mortality of complications after laparoscopic and open distal gastrectomy after a prospective 
Table 4 Subgroup Analysis of Overall Complications

\begin{tabular}{|c|c|c|c|c|c|c|c|c|}
\hline \multirow[t]{2}{*}{ Subgroup } & \multicolumn{2}{|l|}{ OG } & \multicolumn{2}{|l|}{ LG } & \multirow[t]{2}{*}{ OR } & \multicolumn{2}{|l|}{$95 \% \mathrm{Cl}$} & \multirow[t]{2}{*}{$P$} \\
\hline & Total & Rate (\%) & Total & Rate (\%) & & Lower & Upper & \\
\hline Male & 1,303 & 20.9 & 522 & 15.7 & 0.706 & 0.539 & 0.926 & 0.012 \\
\hline Female & 394 & 20.6 & 154 & 14.3 & 0.644 & 0.385 & 1.067 & 0.091 \\
\hline$<60$ & 927 & 18.3 & 373 & 13.3 & 0.705 & 0.502 & 0.990 & 0.043 \\
\hline$\geq 60$ & 770 & 23.8 & 303 & 17.5 & 0.680 & 0.484 & 0.955 & 0.026 \\
\hline $\mathrm{ASA}<\amalg$ & 1,499 & 20.0 & 600 & 14.3 & 0.669 & 0.515 & 0.868 & 0.002 \\
\hline $\mathrm{ASA} \geq_{\amalg}$ & 198 & 26.8 & 76 & 23.7 & 0.849 & 0.459 & I.57| & 0.602 \\
\hline TNM I & 414 & 14.5 & 184 & 10.9 & 0.720 & 0.420 & 1.233 & 0.230 \\
\hline TNM II & 487 & 21.8 & 200 & 16.5 & 0.710 & 0.462 & 1.093 & 0.119 \\
\hline TNM III & 796 & 23.5 & 292 & 17.5 & 0.689 & 0.489 & 0.972 & 0.033 \\
\hline $\mathrm{BMI}<24 \mathrm{~kg} / \mathrm{m}^{2}$ & 1,190 & 20.8 & 475 & 14.1 & 0.624 & 0.465 & 0.837 & 0.002 \\
\hline $\mathrm{BMI} \geq 24 \mathrm{~kg} / \mathrm{m}^{2}$ & 507 & 20.7 & 201 & 18.4 & 0.864 & 0.570 & 1.310 & 0.490 \\
\hline Proximal gastrectomy & 115 & 24.3 & 39 & 10.3 & 0.355 & 0.116 & 1.087 & 0.061 \\
\hline Distal gastrectomy & 692 & 15.5 & 297 & 13.8 & 0.876 & 0.594 & 1.292 & 0.503 \\
\hline Total gastrectomy & 890 & 20.8 & 340 & 15.4 & 0.647 & 0.470 & 0.891 & 0.007 \\
\hline Well-moderate differentiation & 621 & 21.7 & 275 & 18.2 & 0.800 & 0.558 & 1.148 & 0.255 \\
\hline Poor differentiation, Signet ring cell or Mucinous & $\mathrm{I}, 076$ & 20.3 & 401 & 13.5 & 0.612 & 0.443 & 0.846 & 0.003 \\
\hline Tumor size $(<5 \mathrm{~cm})$ & 1,060 & 18.4 & $44 I$ & 16.1 & 0.851 & 0.632 & I.I46 & 0.298 \\
\hline Tumor size $(\geq 5 \mathrm{~cm})$ & 637 & 24.8 & 235 & 14.0 & 0.495 & 0.329 & 0.746 & 0.001 \\
\hline Operation time $<180 \mathrm{~min}$ & 831 & 17.1 & 44 & 13.6 & 0.766 & 0.318 & $\mathrm{I} .847$ & 0.552 \\
\hline Operation time $\geq 180 \mathrm{~min}$ & 866 & 24.4 & 632 & 15.5 & 0.570 & 0.437 & 0.743 & $<0.01$ \\
\hline
\end{tabular}

Table 5 Subgroup Analysis of Severe Complications

\begin{tabular}{|c|c|c|c|c|c|c|c|c|}
\hline \multirow[t]{2}{*}{ Subgroup } & \multicolumn{2}{|l|}{ OG } & \multicolumn{2}{|l|}{ LG } & \multirow[t]{2}{*}{ OR } & \multicolumn{2}{|l|}{$95 \% \mathrm{Cl}$} & \multirow[t]{2}{*}{$P$} \\
\hline & Total & Rate (\%) & Total & Rate (\%) & & Lower & Upper & \\
\hline Male & I,303 & 6.3 & 522 & 5.6 & 0.876 & 0.566 & 1.355 & 0.551 \\
\hline Female & 394 & 4.3 & 154 & 6.5 & 1.540 & 0.689 & 3.442 & 0.289 \\
\hline$<60$ & 927 & 5.2 & 373 & 3.8 & 0.714 & 0.389 & 1.312 & 0.276 \\
\hline$\geq 60$ & 770 & 6.6 & 303 & 8.3 & 1.268 & 0.770 & 2.086 & 0.350 \\
\hline ASA<ய & 1,499 & 5.7 & 600 & 5.5 & 0.968 & 0.640 & $\mathrm{I} .464$ & 0.878 \\
\hline ASA ш & 198 & 5.8 & 76 & 5.8 & I. 127 & 0.416 & 3.048 & 0.814 \\
\hline TNM I & $4 \mid 4$ & 3.9 & 184 & 3.8 & 0.984 & 0.398 & 2.433 & 0.972 \\
\hline TNM II & 487 & 6.0 & 200 & 4.5 & 0.744 & 0.346 & 1.602 & 0.449 \\
\hline TNM III & 796 & 6.8 & 292 & 7.9 & I.175 & 0.707 & 1.952 & 0.533 \\
\hline $\mathrm{BMI}<24 \mathrm{~kg} / \mathrm{m}^{2}$ & 1,190 & 6.3 & 475 & 6.3 & 1.063 & 0.684 & $1.65 \mathrm{I}$ & 0.787 \\
\hline $\mathrm{BMI} \geq 24 \mathrm{~kg} / \mathrm{m}^{2}$ & 507 & 5.5 & 201 & 4.5 & 0.802 & 0.371 & 1.731 & 0.573 \\
\hline Proximal gastrectomy & 115 & 6.1 & 39 & 2.6 & 0.406 & 0.048 & 3.409 & 0.392 \\
\hline Distal gastrectomy & 692 & 3.3 & 297 & 5.1 & 1.547 & 0.796 & 3.009 & 0.195 \\
\hline Total gastrectomy & 890 & 7.8 & 340 & 6.8 & 0.863 & 0.529 & 1.408 & 0.556 \\
\hline Well-moderate differentiation & 621 & 6.1 & 275 & 7.6 & 1.268 & 0.730 & 2.205 & 0.398 \\
\hline Poor differentiation, Signet ring cell or Mucinous & 1,076 & 5.7 & 401 & 4.5 & 0.782 & 0.456 & 1.340 & 0.370 \\
\hline Tumor size $(<5 \mathrm{~cm})$ & 1,060 & 5.0 & 441 & 5.2 & 1.045 & 0.633 & 1.728 & 0.862 \\
\hline Tumor size $(\geq 5 \mathrm{~cm})$ & 637 & 7.2 & 235 & 6.8 & 0.939 & 0.521 & 1.693 & 0.833 \\
\hline Operation time $<180 \mathrm{~min}$ & 831 & 4.2 & 44 & 4.5 & 1.083 & 0.252 & 4.656 & 0.951 \\
\hline Operation time $\geq 180 \mathrm{~min}$ & 866 & 7.8 & 632 & 5.9 & 0.779 & 0.513 & 1.184 & 0.242 \\
\hline
\end{tabular}

randomized controlled study. A systematic review of 13 studies involving a total of 2,794 patients made by British scholars Best et $\mathrm{al}^{16}$ suggested that there were no statistically significant differences in mortality, short-term and long-term results between laparoscopic and open gastrectomy. Lee et $\mathrm{al}^{17}$ studied 1,364 patients with 
Table 6 Univariate and Multivariate Analyses of Postoperative Complications After Radical Gastrectomy

\begin{tabular}{|c|c|c|c|c|c|c|c|c|}
\hline \multirow[t]{2}{*}{ Variables } & \multicolumn{4}{|c|}{ Overall Complications } & \multicolumn{4}{|c|}{ Severe Complications } \\
\hline & $\begin{array}{l}\text { Percent } \\
\text { (\%) }\end{array}$ & Uni-P & $\begin{array}{l}\text { Multi-OR }(95 \% \\
\text { Cl) }\end{array}$ & $\begin{array}{l}\text { Multi- } \\
P\end{array}$ & $\begin{array}{l}\text { Percent } \\
\text { (\%) }\end{array}$ & Uni-P & $\begin{array}{l}\text { Multi-OR (95\% } \\
\text { Cl) }\end{array}$ & $\begin{array}{l}\text { Multi- } \\
P\end{array}$ \\
\hline Age (years) & & 0.002 & & 0.028 & & 0.017 & & 0.037 \\
\hline$<60$ & 17.0 & & 1.000 & & 4.8 & & 1.000 & \\
\hline$\geq 60$ & 22.0 & & $1.269(1.027-1.568)$ & & 7.1 & & $1.449(1.022-2.052)$ & \\
\hline ASA & & 0.003 & & 0.015 & & 0.264 & & \\
\hline$<ш$ & 18.4 & & 1.000 & & 5.6 & & & \\
\hline$\geq_{\amalg}$ & 25.9 & & $1.457(1.077-1.971)$ & & 7.3 & & & \\
\hline Resection extent & & $<0.001$ & & 0.021 & & $<0.001$ & & 0.021 \\
\hline Subtotal gastrectomy & 15.7 & & 1.000 & & 4.0 & & 1.000 & \\
\hline Total gastrectomy & 22.5 & & $1.292(1.040-1.606)$ & & 7.5 & & $1.556(1.068-2.266)$ & \\
\hline Estimate blood loss & & $<0.001$ & & $<0.001$ & & $<0.001$ & & 0.002 \\
\hline$<200 \mathrm{~mL}$ & 14.8 & & 1.000 & & 4.1 & & 1.000 & \\
\hline$\geq 200 \mathrm{~mL}$ & 24.6 & & $1.730(1.402-2.135)$ & & 7.8 & & $1.784(1.248-2.551)$ & \\
\hline P T stage & & $<0.001$ & & $<0.001$ & & 0.002 & & 0.028 \\
\hline $\mathrm{TI}$ & 11.6 & & 1.000 & & 2.8 & & 1.000 & \\
\hline$T 2-4 a$ & 21.1 & & $1.765(1.288-2.418)$ & & 6.6 & & $1.950(1.075-3.537)$ & \\
\hline p N stage & & 0.008 & & & & 0.212 & & \\
\hline No & 16.6 & & & & 5.0 & & & \\
\hline $\mathrm{NI}-3$ & 20.9 & & & & 6.3 & & & \\
\hline Tumor size & & 0.013 & & & & 0.040 & & \\
\hline$<5 \mathrm{~cm}$ & 17.7 & & & & 5.1 & & & \\
\hline$\geq 5 \mathrm{~cm}$ & 21.9 & & & & 7.1 & & & \\
\hline Operation time & & 0.027 & & & & 0.012 & & \\
\hline$<180 \mathrm{~min}$ & 16.9 & & & & 4.2 & & & \\
\hline$\geq 180 \mathrm{~min}$ & 20.6 & & & & 6.7 & & & \\
\hline Operation method & & 0.003 & & & & 0.952 & & \\
\hline LG & 20.8 & & & & 5.8 & & & \\
\hline OG & 15.4 & & & & 5.8 & & & \\
\hline Gender & & 0.754 & & & & 0.311 & & \\
\hline Male & 19.4 & & & & 6.1 & & & \\
\hline Female & 18.8 & & & & 4.9 & & & \\
\hline Histological differentiation & & 0.181 & & & & 0.212 & & \\
\hline Well-Moderate differentiation & 20.6 & & & & 6.6 & & & \\
\hline Poor differentiation, Signet ring cell or & 18.4 & & & & 5.3 & & & \\
\hline Mucinous & & & & & & & & \\
\hline BMI & & 0.520 & & & & 0.424 & & \\
\hline$<24 \mathrm{~kg} / \mathrm{m}^{2}$ & 18.9 & & & & 6.1 & & & \\
\hline$\geq 24 \mathrm{~kg} / \mathrm{m}^{2}$ & 20.1 & & & & 5.2 & & & \\
\hline Number of retrieved & & 0.336 & & & & 0.740 & & \\
\hline$<25$ & 18.5 & & & & 6.0 & & & \\
\hline$\geq 25$ & 20.0 & & & & 5.7 & & & \\
\hline Number of retrieved lymph & & 0.153 & & & & 0.754 & & \\
\hline
\end{tabular}

Note: P-values less than 0.05 are highlighted in bold.

Abbreviations: Uni, univariate logistic regression analysis; Multi, multivariate logistic regression analysis; OR, odd ratio; $\mathrm{Cl}$, confidence interval. 
laparotomy and laparoscopy-assisted distal gastrectomy, and found that the incidence of overall complications (25.3\% vs $40.1 \%, P=0.001)$ and severe complications $(2.1 \%$ vs $5.4 \%, P=0.001)$ in the laparoscopic group was relatively lower than that of open gastrectomy. Metaanalysis by Zou et $\mathrm{al}^{18}$ also found that, although laparoscopic surgery has higher technical requirements and longer operation time, the incidence of postoperative complications was lower than laparotomy. In the study of 4,116 patients, Higgins et $\mathrm{al}^{19}$ believed that, compared with laparotomy, there were fewer complications in the laparoscopic group $(\mathrm{OR}=0.61,95 \% \quad \mathrm{CI}=0.45-0.82$, $P=0.001)$, and no difference in mortality $(\mathrm{OR}=0.74,95 \%$ $\mathrm{CI}=0.32-1.72 . \quad P=0.481)$. However, Korean scholars Yasunaga et $\mathrm{al}^{20}$ studied 2,473 pairs of patients after propensity score matching with distal gastrectomy of early gastric cancer and found that laparoscopic surgery could slightly shorten the postoperative hospitalization time (13 days vs 15 days, $P<0.001$ ), without affecting the incidence of postoperative complications ( $12.9 \%$ vs $12.6 \%, P=0.73$ ) and the hospital mortality $(0.36 \%$ vs $0.28 \%, P=0.80)$. Japanese scholars Shinohara et $\mathrm{al}^{21}$ analyzed 336 patients with advanced gastric cancer and found that there were no statistically significant differences in the incidence of postoperative complications $(24.2 \%$ vs $28.5 \%, P=0.402)$, mortality $(1.1 \%$ vs $0, P=0.519)$, and 5 -year survival rate $(62.0 \%$ vs $63.7 \%, P=0.968)$ between the laparoscopy and laparotomy groups. Tu et $\mathrm{al}^{22}$ analyzed 4,124 patients and concluded that there was no statistically significant difference in the incidence of complications between laparoscopy and laparotomy ( 14.2 vs $16.5 \%, P=0.093)$, but the mortality rate of patients with laparotomy was higher $\mathrm{n}$ the case of complications $(0.3 \%$ vs $1.2 \%, P=0.004)$. Huscher et $\mathrm{al}^{23}$ considered that there was no difference in mortality (3.3\% vs $6.7 \%)$ or the complication rate $(27.6 \%$ vs $26.7 \%)$ after laparoscopic and open gastrectomy, but the laparoscopy group had less estimated blood loss, earlier postoperative oral intake, and shorter hospitalization time.

As with open surgery, the basic criteria for laparoscopic radical gastrectomy are proper perigastric lymph node dissection and R0 resection. This study suggests that laparoscopy could achieve a lower number of lymph nodes retrieved than that in open surgery. The reasons for this were related to the experience of the surgeon, the poor location of lymph nodes in actual lymph node dissection, and the occlusion of the surgical field. While some studies suggested that there was no significant difference in the number of lymph nodes removed between the two operations for Shuang et al, ${ }^{5} \mathrm{Hu}$ et al, ${ }^{24}$ and Parisi et al. ${ }^{25}$ Different from the researchof Lee et al, ${ }^{17}$ Yasunaga et al, ${ }^{20}$ and Huscher et al, ${ }^{23}$ this study found that the overall complication rate of patients in the LG group was statistically lower than in the OG group $(15.4 \%$ vs $20.8 \%$, $\mathrm{P}=0.003$ ), however, no difference was found in the incidence of severe complications ( $5.8 \%$ vs $5.8 \%, P=0.952)$.

The incidence of mild complications in the LG group was significantly lower than in the OG group, which was mainly reflected in the significantly lower incidence of mild wound complications, pulmonary infection, which may be contributed to the minute incision, less postoperative pain, and beneficial to postoperative cough and sputum drainage of patients. This result was approved by the study of Shinohara et al. ${ }^{21}$ Subgroup analysis was conducted according to gender, age, ASA classification, TNM stage, histologic type, gastric resection extent, and tumor size, and operation time showed the same pattern in most stratifications. Multivariate analysis revealed that laparoscopic surgery is a protective factor for the reduction of postoperative complications, age $\geq 60$ years, ASA classification $\amalg$, and estimated blood loss $\geq 200 \mathrm{~mL}$ were confirmed as independent risk factors of overall complications. Age $\geq 60$ years, tumor stage T2-T4a, estimated blood loss $\geq 200 \mathrm{~mL}$ were confirmed as independent predictors for severe complications.

First, abdominal hemorrhage was a serious complication and an important cause of conversion from laparoscopic surgery to open surgery, including intraoperative and postoperative bleeding. The operator should have rich experience in laparotomy, and be familiar with the anatomical positioning marks and the changes of visual field under laparoscopy. It can greatly reduce the bleeding rate if the lymph nodes are free from the special fascia space during lymph node dissection; at the same time, mastering the essentials of electrocoagulation hemostasis with an ultrasonic scalpel could improve the safety of hemostasis. Second, the causes of postoperative intestinal obstruction were complex, which may be related to adhesion and inflammatory reaction. Careful bowel preparation should be carried out before operation, reducing the risk of contamination caused by traction and incision of intestinal tube during operation, controlling abdominal inflammatory reaction, completing hemostasis of wound surface, and early guiding patients to get out of bed after operation, which is conducive to the recovery of gastrointestinal function. Third, anastomotic complications included anastomotic bleeding, anastomotic stenosis, and anastomotic 
leakage, which were related to improper use of a stapler. Skilled use of the stapler can reduce the occurrence of such complications.

This study found that, compared with open gastrectomy patients, the operation time of laparoscopic gastrectomy was significantly longer, but it had the advantage of being minimally invasive. This study found that the laparoscopic surgery method involved less estimated blood loss and reduced overall postoperative complications. Moreover, academics have already confirmed that, with the development of the laparoscopic technique, and increased experience of the surgeons, the operation time can be greatly reduced. ${ }^{26,27}$ This study focused on the incidence of complications, specific types, and possible risk factors of LG and OG for radical resection of early gastric cancer. Through this retrospective and large sample cohort study, we believed that the data and conclusion were more objective and convincing. We should also see that, before matching, the two groups had many great differences including the scope of gastrectomy, the mode of digestive tract reconstruction, the degree of tumor pathological differentiation, and the degree of tumor pathological differentiation, the depth of invasion, the lymph node metastasis, the TNM stage, the tumor length, and so on, which were often important adverse factors to affecting the occurrence and severity of postoperative complications. Therefore, we still cannot conclude that the risk factors screened in this study are comprehensive enough. Of course, we also think that the experience of the operator, the basic state of the patient's whole body, and nursing management ability also have an important influence on the occurrence of complications.

Clavien-Dindo classification standard plays an important role in the evaluation of postoperative complications in gastric cancer. This study compared the overall complications and severe complications of matched LG and OG groups, and carried out univariate and multivariate regression analysis. Different from previous studies, the feature of this study was to compare the incidence of complications through large sample data, and provide a more real, comprehensive clinical world.

In conclusion, compared with traditional open gastrectomy, laparoscopic radical gastrectomy is safe and feasible with a better minimally invasive effect for the treatment of gastric cancer in terms of the lower incidence of overall complications.

\section{Abbreviations}

LG, laparoscopic gastrectomy; OG, open gastrectomy; ASA, American Society of Anesthesiologists; BMI, Body mass index; $\mathrm{T}$, tumor; $\mathrm{N}$, node; $\mathrm{M}$, metastasis; $\mathrm{TNM}$, Tumor-node metastases; SD, standard deviation; OR, odd ratio; CI, Confidence Interval.

\section{Data Sharing Statement}

The datasets analyzed during the current study are available from the corresponding author on reasonable request.

\section{Ethics Approval and Consent to Participate}

This study was conducted in accordance with the Declaration of Helsinki and approved by the XiJing Ethical Review Committee. Written informed consent was obtained from all the patients prior to surgery.

\section{Acknowledgments}

The authors are thankful to the medical staff of Xijing Hospital of Digestive Diseases for their management of the database.

\section{Disclosures}

Drs. Bo Lian, Jie Chen, Zhengyan LI, Gang Ji, Shiqi Wang, Qingchuan Zhao, and Mengbin Li have no conflicts of interest or financial ties to disclose.

\section{Funding}

This study was Supported by the Funding program: Shan Xi Natural Science Basic Research Program project (2017JM8026) and Air Force Military Medical University Technology Development Funded project (2019XC029). The funding body had no role in the design of the study and collection, analysis, and interpretation of data and in writing of this manuscript.

\section{References}

1. Wang F, Shen L, Li J, et al. The Chinese society of clinical oncology (CSCO): clinical guidelines for the diagnosis and treatment of gastric cancer. Cancer Commun (Lond). 2019;39(1):10. doi:10.1186/s40880-019-0349-9

2. Dindo D, Demartines N, Clavien P-A. Classification of surgical complications a new proposal with evaluation in a cohort of 6336 patients and results of a survey. Ann Surg. 2004;240(2):205-213. doi:10.1097/ 01.sla.0000133083.54934.ae

3. Sano T, Coit D, Kim H, et al. Proposal of a new stage grouping of gastric cancer for TNM classification: international gastric cancer association staging project. Gastric Cancer. 2017;20(2):217-225. doi:10.1007/s10120-016-0601-9 
4. Japanese Gastric Cancer Association. Japanese gastric cancer treatment guidelines 2014 (ver. 4). Gastric Cancer. 2017;20(1):1-19. doi:10.1007/s10120-016-0622-4

5. Shuang J, Sheng B, Zheng J, et al. A case-control study of laparoscopy-assisted and open distal gastrectomy for advanced gastric cancer. J Gastrointest Surg. 2011;15(1):57-62. doi:10.1007/s11605010-1361-1

6. Fang C, Hua J, Li J, et al. Comparison of long-term results between laparoscopy-assisted gastrectomy and open gastrectomy with D2 lymphad-enectomy for advanced gastric cancer. Am J Surg. 2014;208 (3):391-396.

7. Baiocchi G, Giacopuzzi S, Marrelli D, et al. International consensus on a complications list after gastrectomy for cancer. J Gastric Cancer. 2019;22(1):172-189. doi:10.1007/s10120-018-0839-5

8. Clavien P, Barkun J, de Oliveira ML. The Clavien-Dindo classification of surgical complications: five-year experience. J Ann Surg. 2009;250(2):187-196. doi:10.1097/SLA.0b013e3181b13ca2

9. Li Z, Ji G, Bai B, et al. Laparoscopy-assisted distal gastrectomy versus laparoscopy-assisted total gastrectomy with D2 lymph node dissection for middle-third advanced gastric cancer. J Surg Endosc. 2018;32(5):2255-2262. doi:10.1007/s00464-017-5919-9

10. Yu J, Ji J, Li G, et al. Effect of laparoscopic vs open distal gastrectomy on 3-year diseasefree survival in patients with locally advanced gastric cancer the class-01 randomized clinical trial. JAMA. 2019;321 (20):1983-1992.

11. Li Z, Zhao Q, Bai B, et al. Enhanced recovery after surgery programs for laparoscopic abdominal surgery: a systematic review and meta-analysis. World J Surg. 2018;42(11):3463-3473. doi:10.1007/ s00268-018-4656-0

12. Cheng Q, Jiang C, Ge S. Selection of digestive tract reconstruction in laparoscopic radical gastrectomy for gastric cancer. Chin J Laparosc Surg. 2016;9(01):55-58.

13. Katayama H, Kurokawa Y, Nakamura K, et al. Extended Clavien-Dindo classification of surgical complications: Japan clinical oncology group postoperative complications criteria. J Surg Today. 2016;46(6):668-685. doi:10.1007/s00595-015-1236-x

14. Bolliger M, Kroehnert J, Molineus F, et al. Experiences with the standardized classification of surgical complications (Clavien-Dindo) in general surgery patients. Eur Surg. 2018;50 (6):256-261. doi:10.1007/s10353-018-0551-z

15. Kim H, Han S, Kim M. Long-term results of laparoscopic gastrectomy for gastric cancer: a large scale case-control and case-matched Korean multicenter study. J Clin Oncol. 2014;32(7):627-633. doi:10.1200/JCO.2013.48.8551
16. Best L, Mughal M, Gurusamy K, et al. Laparoscopic versus open gastrectomy for gastric cancer. Cochrane Database Syst Rev. 2016;31 (3):CD011389.

17. Lee J, Park D, Kim H, Lee H-J, Yang H-K. Comparison of complications after laparoscopy-assisted distal gastrectomy and open distal gastrectomy for gastric cancer using the Clavien-Dindo classification. Surg Endosc. 2012;26(5):1287-1295. doi:10.1007/s00464-0112027-0

18. Zou Z, Zhao L, Mou T, et al. Laparoscopic vs open D2 gastrectomy for locally advanced gastric cancer: a meta-analysis. World $J$ Gastroenterol. 2014;20(44):16750-16764. doi:10.3748/wjg.v20. i44.16750

19. Higgins R, Kubasiak J, Jacobson R, et al. Outcomes and use of laparoscopic versus open gastric resection. JSLS. 2015;19(4):95. doi:10.4293/JSLS.2015.00095

20. Yasunaga H, Horiguchi H, Kuwabara K, et al. Outcomes after laparoscopic or open distal gastrectomy for early-stage gastric cancer. Ann Surg. 2013;257(4):640-646. doi:10.1097/SLA.0b013e31826fd541

21. Shinohara T, Satoh S, Kanaya S, et al. Laparoscopic versus open D2 gastrectomy for advanced gastric cancer: a retrospective cohort study. Surg Endosc. 2013;27(1):286-294. doi:10.1007/s00464-012-2442-x

22. Tu R, Lin J, Zheng $\mathrm{C}$, et al. Complications and failure to rescue following laparoscopic or open gastrectomy for gastric cancer: a propensity-matched analysis. Surg Endosc. 2017;31(5):2325-2337. doi:10.1007/s00464-016-5235-9

23. Huscher C, Mingoli A, Sgarzini G, et al. Laparoscopic versus open subtotal gastrectomy for distal gastric cancer. Ann Surg. 2005;241 (2):232-237.

24. Hu Y, Huang C, Sun Y, et al. Morbidity and Mortality of laparoscopic versus open D2 distal gastrectomy for advanced gastric cancer: a randomized controlled trial. $J$ Clin Oncol. 2016;34 (12):1350-1357. doi:10.1200/JCO.2015.63.7215

25. Parisi A, Reim D, Borghi F, et al. Minimally invasive surgery for gastric cancer: a comparison between robotic, laparoscopic and open surgery. World J Gastroenterol. 2017;23(13):2376-2384. doi:10.3748/wjg.v23.i13.2376

26. Kunisaki C, Makino H, Yamamoto N, et al. Learning curve for laparoscopy-assisted distal gastrectomy with regional lymph node dissection for early gastric cancer J. Surg Laparosc Endosc Percutan Tech. 2008;18(3):236-241. doi:10.1097/ SLE.0b013e31816aa13f

27. Huang C, Chen Q, Lin J, et al. Huang's three-step maneuver for laparoscopic spleen-preserving No.10 lymph node dissection for advanced proximal gastric cancer. Chin J Cancer Res. 2014;26 (2):208-210
Cancer Management and Research

\section{Publish your work in this journal}

Cancer Management and Research is an international, peer-reviewed open access journal focusing on cancer research and the optimal use of preventative and integrated treatment interventions to achieve improved outcomes, enhanced survival and quality of life for the cancer patient.
The manuscript management system is completely online and includes a very quick and fair peer-review system, which is all easy to use Visit http://www.dovepress.com/testimonials.php to read real quotes from published authors. 